\title{
The fabrication and properties of magnetorheological elastomers employing bio-inspired dopamine modified carbonyl iron particles
}

\author{
Yanfen Zhou \\ Qingdao University \\ Lele Li \\ Qingdao University \\ Wenyue Li \\ Qingdao University
}

See next page for additional authors

Follow this and additional works at: https://arrow.tudublin.ie/cerart

Part of the Materials Chemistry Commons, and the Polymer and Organic Materials Commons

\section{Recommended Citation}

Yanfen Zhou et al 2020 Smart Mater. Struct. 29055005

This Article is brought to you for free and open access by the Centre for Elastomer Research at ARROW@TU Dublin. It has been accepted for inclusion in Articles by an authorized administrator of ARROW@TU Dublin. For more information, please contact arrow.admin@tudublin.ie, aisling.coyne@tudublin.ie, gerard.connolly@tudublin.ie.

Funder: National Natural Science Foundation of China; Shandong Provincial Natural Science Foundation, China; Qingdao Postdoctoral Applied Basic Research Project

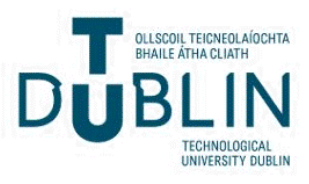




\section{Authors}

Yanfen Zhou, Lele Li, Wenyue Li, Shipeng Wen, Liang Jiang, Stephen Jerrams, Jianwei Ma, and Shaojuan Chen 


\section{PAPER}

The fabrication and properties of magnetorheological elastomers employing bio-inspired dopamine modified carbonyl iron particles

To cite this article: Yanfen Zhou et al 2020 Smart Mater. Struct. 29055005

View the article online for updates and enhancements. 


\title{
The fabrication and properties of
}

\section{magnetorheological elastomers employing bio-inspired dopamine modified carbonyl iron particles}

\author{
Yanfen $\mathrm{Zhou}^{1}$, Lele $\mathrm{Li}^{1}$, Wenyue $\mathrm{Li}^{1}$, Shipeng Wen ${ }^{2}$, Liang Jiang ${ }^{1}{ }^{(1)}$, \\ Stephen Jerrams ${ }^{3}$, Jianwei Ma ${ }^{1}$ and Shaojuan Chen ${ }^{1}$ \\ ${ }^{1}$ College of Textiles and Clothing, Qingdao University, Qingdao 266071, People's Republic of China \\ ${ }^{2}$ Beijing Engineering Research Center of Advanced Elastomers, Beijing University of Chemical \\ Technology, Beijing 100029, People's Republic of China \\ ${ }^{3}$ Technological University Dublin (TUD), Ireland \\ E-mail: liang.jiang@qdu.edu.cn
}

Received 24 November 2019, revised 29 January 2020

Accepted for publication 20 February 2020

Published 26 March 2020

\begin{abstract}
To obtain magnetorheological elastomers (MREs) with improved mechanical properties and exhibiting an enhanced magnetorheological (MR) effect, bio-inspired dopamine modification has been used to improve the functionality at the surface of carbonyl iron (CI) particles. Various techniques including x-ray photoelectron spectroscopy (XPS), scanning electron microscopy (SEM) and transmission electron microscopy (TEM) were used to confirm that a polydopamine (PDA) layer of about $27.5 \mathrm{~nm}$ had been successfully deposited on the surface of the carbonyl iron particles prior to their inclusion in the MRE composites. The magnetic properties of PDA modified CI particles were shown to be almost the same as those for untreated CI particles. With the introduction of a PDA layer to the surfaces of the particles, both the tensile strength and the elongation at break of the MREs were improved. Furthermore, the MRE composites filled with PDA-coated CI particles exhibited lower zero-field storage moduli but higher magnetic field induced storage moduli when magnetization saturation was reached. The absolute and relative MR effect for the MREs reached $0.68 \pm 0.002 \mathrm{MPa}$ and 294\% respectively, which were higher than those of MREs with pristine CI particles whose absolute and relative MR effect were $0.57 \pm 0.02 \mathrm{MPa}$ and $187 \%$ respectively. The findings of this work provide insights into enhanced fabrication of MREs with both improved mechanical properties and magneto-induced performance.
\end{abstract}

Keywords: mangetorheological elastomers, carbonyl iron, dopamine, surface modification, shear modulus

(Some figures may appear in colour only in the online journal)

\section{Introduction}

Smart materials, also known as stimuli-responsive materials, have attracted tremendous attention in recent years for their wide potential applications in numerous fields. Mangetorheological elastomers (MREs) are a range of smart materials that can change their viscoelastic properties when exposed to external magnetic fields [1]. Tunable viscoelastic changes in modulus make MREs suitable materials for many applications such as vibration absorbers [2-5], adaptive dampers [6] and sensors [7, 8].

MREs consist of soft ferromagnetic particles suspended in elastomeric matrices. The magnetic particles are key in determining the performance of MREs. It has been shown that 
the type of magnetic particles, the particle concentration, their size and shape, magnetic properties and spatial distribution within the matrix all have significant influence on the fieldinduced properties of MREs [9-16]. As well as particlerelated factors, the selection of a suitable matrix materials also plays an important role in determining their effectiveness [17-19]. Generally, the matrix should be highly elastic to allow the rearrangement of the particles under a magnetic field, and thereby to enhance the magnetic field induced mechanical properties of MREs. Consequently, silicone rubber and polyurethane, both with high elasticity, have been widely used as the matrix materials to fabricate various MRE composites [20-22]. In order to meet the likely requirement for mechanical strength in practical engineering applications, natural rubber, styrene-butadiene rubber and various polymer blends have also been used to fabricate MREs with improved mechanical properties [23-26].

Since MREs are polymer based composite material, their performance is also influenced by the quality of the interface between magnetic particles and the elastomeric matrix $[27,28]$. Often, the introduction of inorganic particles into the polymer matrix leads to weak interfacial interaction between the two components, especially when a high content of magnetic particles (normally above $50 \%$ by weight) is incorporated to achieve a desirable magnetorheological (MR) effect. In addition, particle agglomerations is usually formed due to the Van der Waals forces between magnetic particles. Various methods including polymer coating [29-31] and bifunctional coupling agent treatments [32, 33] have been reported to modify the surface of magnetic particles with the aim of improving their bonding with the elastomeric matrix. For example, Yu et al [29] prepared an MRE containing polyaniline (PANI)-modified carbonyl iron particles; the results showed that the PANI modification enhanced the interfacial interaction of the MRE. The MRE sample with PANI-modified CIPs exhibited a larger storage modulus, smaller loss factor and a lower magnitude of the Payne effect. Cvek et al [30] grafted the carbonyl iron particles with poly (trimethylsilyloxyethyl methacrylate) (PHEMATMS) through surface-initiated atom transfer radical polymerization. It was found that the PHEMATMS grafts improved the particle filler/polydimethylsiloxane matrix interactions and the MR effect of the MRE. Although to a certain extent these methods can improve the interfacial interactions and the performance of MREs, they have practical limitations, such as a need for multi-step production, the use of complex instruments and environmental challenges. Hence, finding a new method which is simple, efficient, nontoxic, and easy to control becomes more and more important in order the quest for researchers to improve the performance of MREs.

Inspired by the strong adhesion of mussels, wherein long-lasting adhesion in a wet environment and adherence to almost all types of organic and inorganic surfaces is achieved, Lee et al successfully coated poly(dopamine) (PDA) on the surface of $\mathrm{SiO}_{2}, \mathrm{TiO}_{2}, \mathrm{Cu}$, and $\mathrm{Au}$ in 2007 [34]. Because of its simple ingredients, mild reaction conditions and non-polluting properties, the self-polymerization of dopamine provides a convenient and effective method for surface coating and adhesion purposes, and has been used widely for the surface modification of various organic/inorganic materials [35-37]. However, improvement of interfacial interactions and magnetic field induced viscoelastic properties of MREs through PDA functionalization has not been reported on to date.

In this work, the surface of carbonyl iron particles was modified through self-polymerization of dopamine and the effect of dopamine modification on the surface morphology and magnetic properties of the carbonyl iron particles was evaluated. The influence of the polydopamine surface modification on the microstructure, mechanical properties and magnetic field induced viscoelastic properties of silicone rubber based MREs was also investigated.

\section{Experimental}

\subsection{Materials}

Two-component room temperature vulcanized (RTV) silicone rubber (SR) was purchased from the Red Leaves Company, Shenzhen, China. Soft carbonyl iron (CI) particles with size distributions of $\mathrm{d} 10=3.6 \mu \mathrm{m}, \mathrm{d} 50=7.3 \mu \mathrm{m} \quad$ and $\mathrm{d} 90=16.8 \mu \mathrm{m}$ (Grade CN) were supplied by BASF, Germany. Dopamine hydrochloride (DA.HCl), tris (hydroxymethyl) aminomethane (Tris) and ethanol were procured from Macklin, China.

\subsection{Surface modification of $\mathrm{Cl}$ particles}

Initially, the surface of CI particles was activated by treating them with ethanol for $20 \mathrm{~min}$. Then, the surface activated CI particles were functionalized with polydopamine (PDA). Briefly, DA solution $(400 \mathrm{ml})$ with a concentration of $2.0 \mathrm{~g} \cdot \mathrm{L}^{-1}$ was prepared by dissolving $\mathrm{DA} \cdot \mathrm{HCl}$ powder in distilled water and the $\mathrm{pH}$ value of the solution was adjusted to 8.5 by adding Tris. Pre-treated CI particles $(10 \mathrm{~g})$ were then put in the DA solution and processed at $25^{\circ} \mathrm{C}$ for $24 \mathrm{~h}$. After modification, the CI particles were separated from the solution with a magnet, rinsed with distilled water three times and then dried in a vacuum oven at $60{ }^{\circ} \mathrm{C}$ overnight. Surface modified CI particles have been denoted as CI-PDA in the following text.

\subsection{Fabrication of MREs}

Anisotropic silicone rubber based MREs were fabricated by incorporating $60 \mathrm{wt} \%$ of either CI or CI-PDA particles into the two-component silicone rubber. Firstly, the particles were added to part A of the RTV SR and mechanically stirred for $10 \mathrm{~min}$. Then, part B of the RTV SR was added to the mixture and mechanically stirred for another $10 \mathrm{~min}$. The resulting mixture was poured into a mold and degassed in a vacuum to remove entrapped bubbles. Finally, the whole mixture was placed in an electromagnet and cured under a magnetic flux density of $500 \mathrm{mT}$ for $2 \mathrm{~h}$. The MRE samples obtained, containing either CI or CI-PDA particles were denoted as $\mathrm{SR} / \mathrm{CI}$ and SR/CI-PDA respectively. The surface modification 


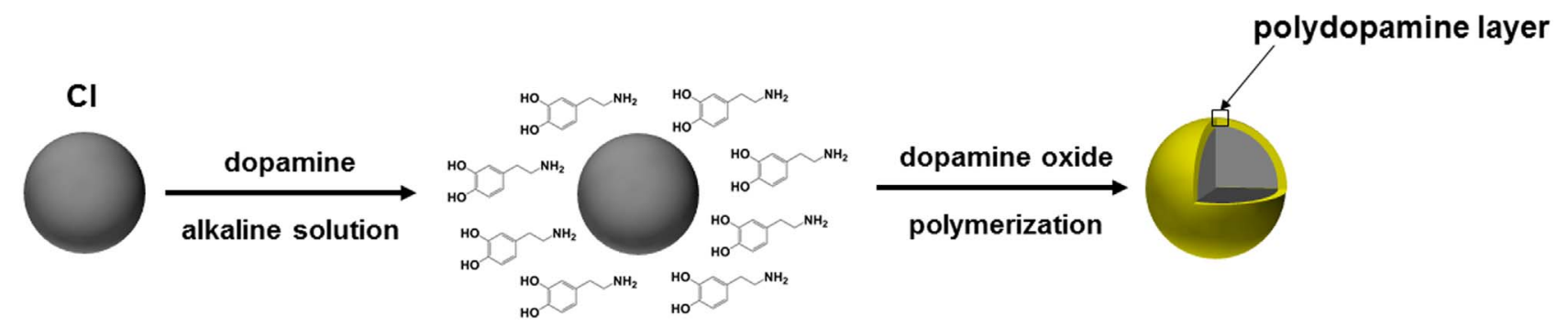

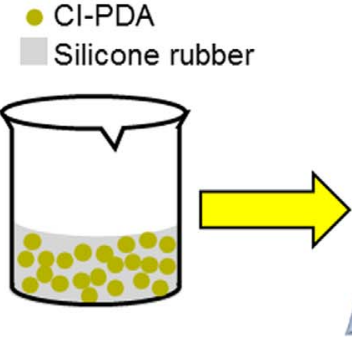

Mixing

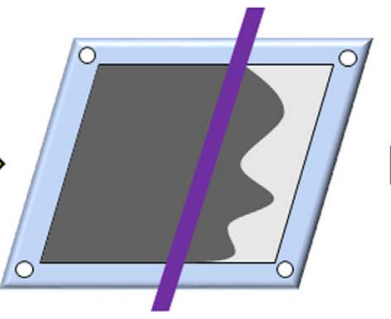

Molding
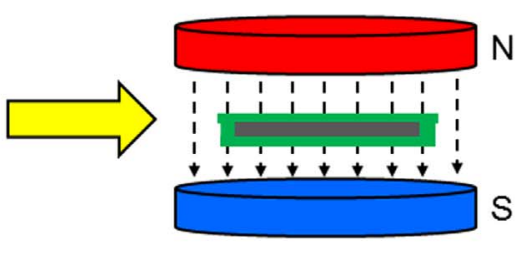

Pre-structuring
MRE
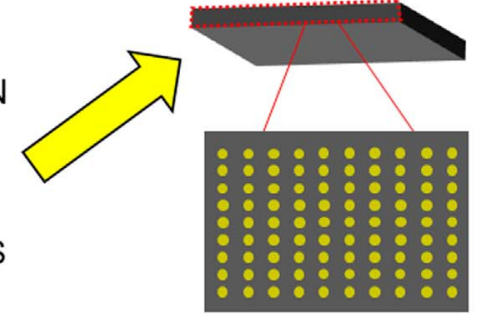

Curing

Figure 1. Schematic illustration of the procedure for surface modification of CI particles and preparation of MREs.

process of CI particles and the preparation of MREs are schematically illustrated in figure 1 .

\subsection{Characterization}

The chemical compositions of the surfaces of the CI and CIPDA microparticles were determined by x-ray photoelectron spectroscopy (XPS). XPS measurements were carried out on an ESCALAB 250XI XPS system (Thermo Electron Corporation, USA) with an $\mathrm{Al} \mathrm{Ka}$ x-ray source $(1486.6 \mathrm{eV}$ photons). The core-level signals were obtained at a photoelectron take-off angle of $45^{\circ}$ with respect to the sample surface.

The magnetic properties ( $M-H$ curve) of the pure CI and CI-PDA particles were measured by using a physical property measurement system (PPMS-9, Quantum Design, America). Measurements were carried out at a temperature of $300 \mathrm{~K}$.

Surface morphologies of the CI and CI-PDA particles were observed by using a scanning electron microscope (SEM) (VEGA3, TESCAN, Czech) operating at a voltage of $10 \mathrm{kV}$ and a high-resolution transmission electron microscope (HR-TEM) (JEM2100F, JEOL, Japan) operating at a voltage of $200 \mathrm{kV}$. The morphologies of the MREs filled with CI and CI-PDA particles were investigated by SEM using the Hitachi VEGA3 scanning electron microscope. The distribution of iron $(\mathrm{Fe})$ in the MRE composites was mapped by using an energy dispersive $\mathrm{X}$-ray spectrometer (EDX) (E1856-C2B, EDAX, USA).

The MR behavior and dynamic viscoelastic properties of MRE specimens were tested in shear oscillation mode using an advanced commercial rheometer (MCR302, Anton Paar, Austria) equipped with an electromagnet (MRD 170). The magnetic flux density was varied from 0 to approximately $1 \mathrm{~T}$ by altering the driving current in the coil of the electromagnet in the range of $0-5 \mathrm{~A}$. The specimens were prepared in disc form with $20 \mathrm{~mm}$ diameter and $1 \mathrm{~mm}$ thickness. During the testing process, the samples were held between a stationary lower plate and an upper plate connected to a rotor subject to forced torsion oscillations.

\section{Results and discussion}

\subsection{Dopamine self-polymerization on the surface of $\mathrm{Cl}$ particles}

The presence of PDA on the surface of CI particles was determined by using XPS, SEM, and HR-TEM. Figure 2(a) shows the XPS wide-scan spectra of CI and CI-PDA particles. The peak at $780 \mathrm{eV}$ represents the occurrence of Fe. The appearance of the peak at $529.3 \mathrm{eV}$ for oxygen $(\mathrm{O})$ in the spectrum of CI particles might be due to the presence of a small amount of hydroxyl groups on the metal surface [30]. The small amount of carbon $(283.8 \mathrm{eV})$ can be considered as an impurity. Compared with CI particles, the spectrum of CIPDA particles show the signal from the $\mathrm{N}$ 1s electron of the dopamine on the CI-PDA particles. The $\mathrm{N} 1 \mathrm{~s}$ core-level spectrum of the CI-PDA particles shown in figure 2(b) can be curve-fitted with two peak components; one peak at $399.5 \mathrm{eV}$ attributed to the amine $(-\mathrm{N}-\mathrm{H})$ groups and the other at $398.5 \mathrm{eV}$ attributed to the imine $(-\mathrm{N}=)$ groups. The amine $(-\mathrm{N}-\mathrm{H})$ groups belong to dopamine, while the imine $(-\mathrm{N}=)$ groups are formed by the indole groups through structure evolution during the dopamine self-polymerization [38]. The XPS results suggest that PDA successfully deposited on the surface of CI particles through the oxidative self-polymerization of dopamine.

SEM was used to observe the surface topography of CI and CI-PDA particles, and the results are shown in figures 3(a) and (b). It can be observed that the surfaces of 

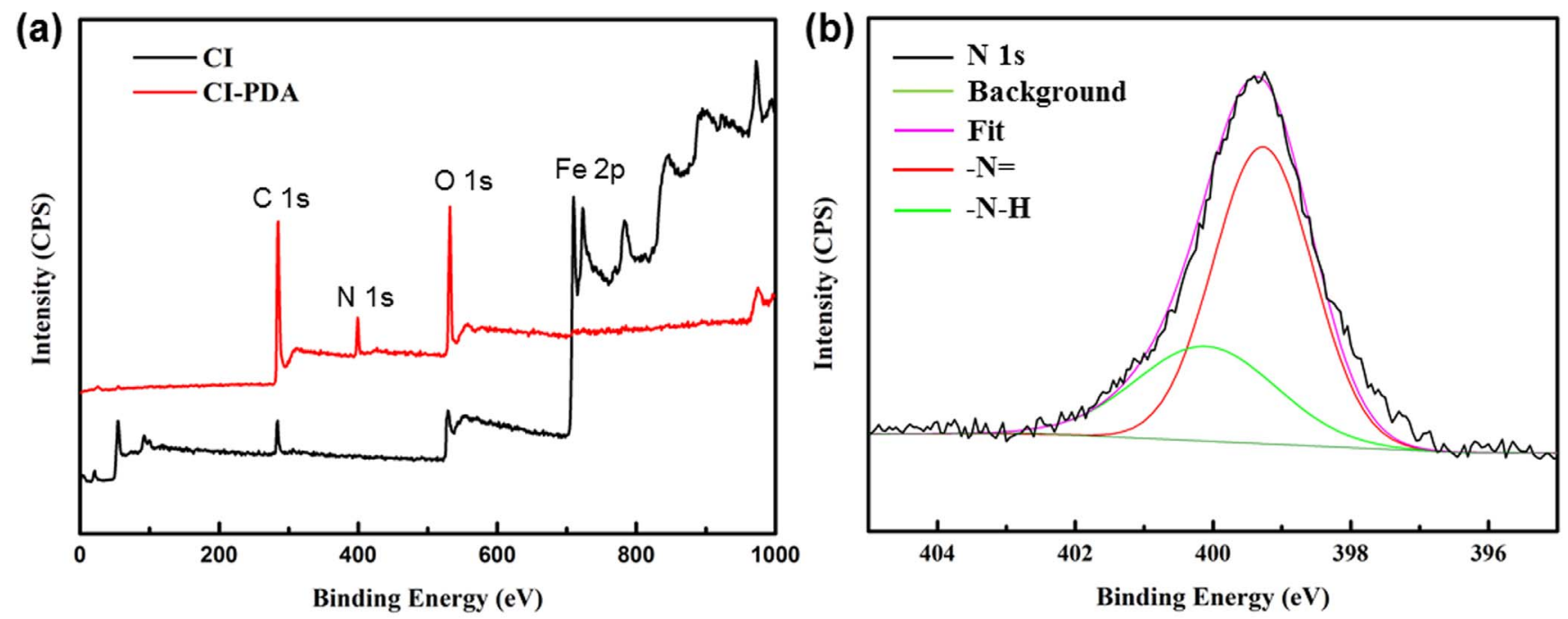

Figure 2. (a) X-ray photoelectron spectra of CI and CI-PDA particles and (b) the $\mathrm{N}$ 1s core-level spectrum of the CI-PDA particles.
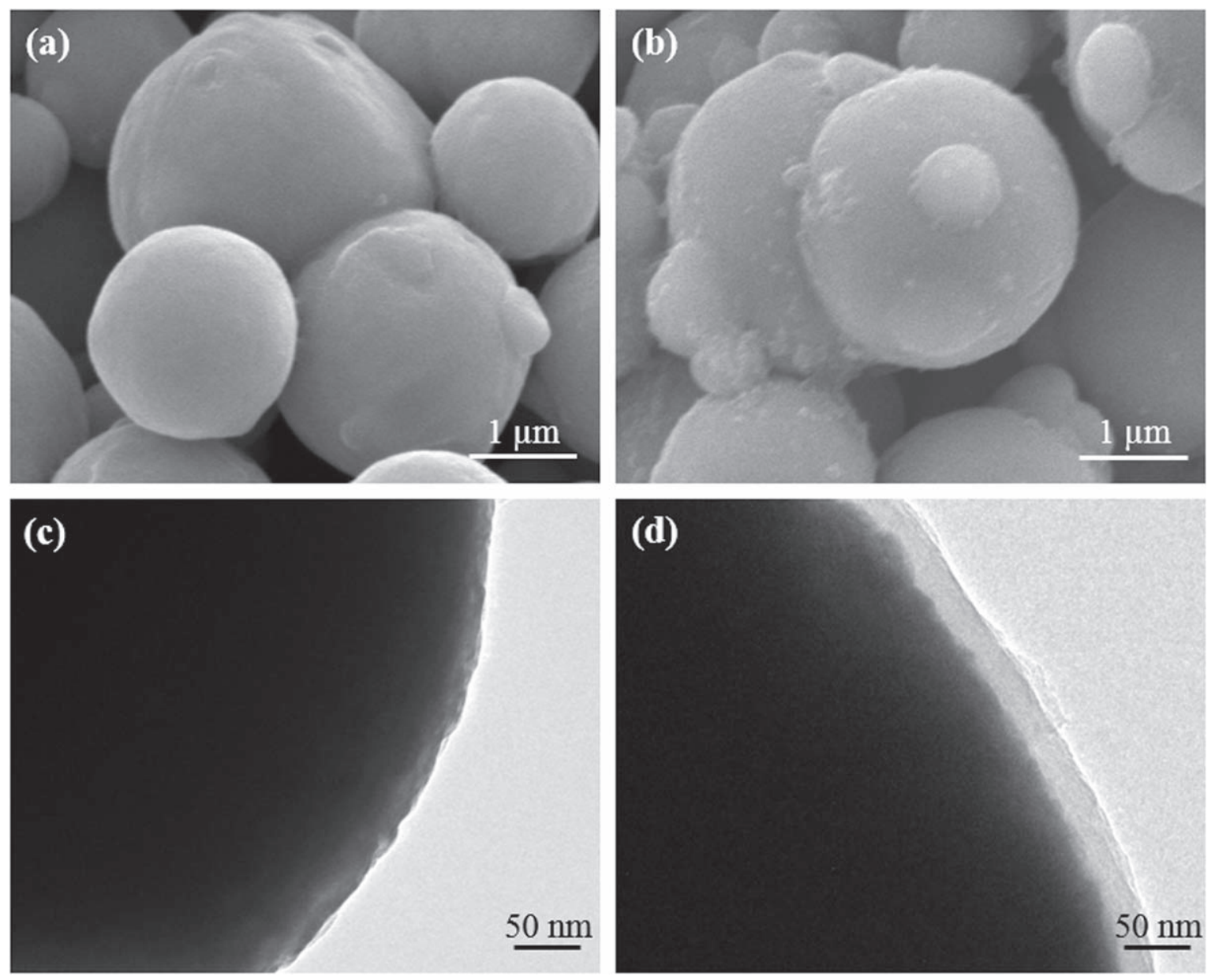

Figure 3. SEM micrographs of (a) CI particles and (b) CI-PDA particles and HR-TEM images of (c) CI particles and (d) CI-PDA particles.

CI-PDA particles were rougher than those of the CI particles, probably because of the deposited polydopamine on the surface of the CI particles. Moreover, the distinct layer of PDA observed by HR-TEM, as shown in figures 3(c) and (d), further confirmed the successful deposition of PDA on the CI particles. From figure $3(\mathrm{~d})$ it can be observed that the thickness of the PDA coating on the CI particles was approximately $27.5 \mathrm{~nm}$.

The magnetic properties of the untreated CI and CI-PDA were investigated and the $M-H$ curves are shown in figure 4. Both kinds of particles exhibited almost hysteresis-free loops, small coercivities and remanent magnetizations, indicating 


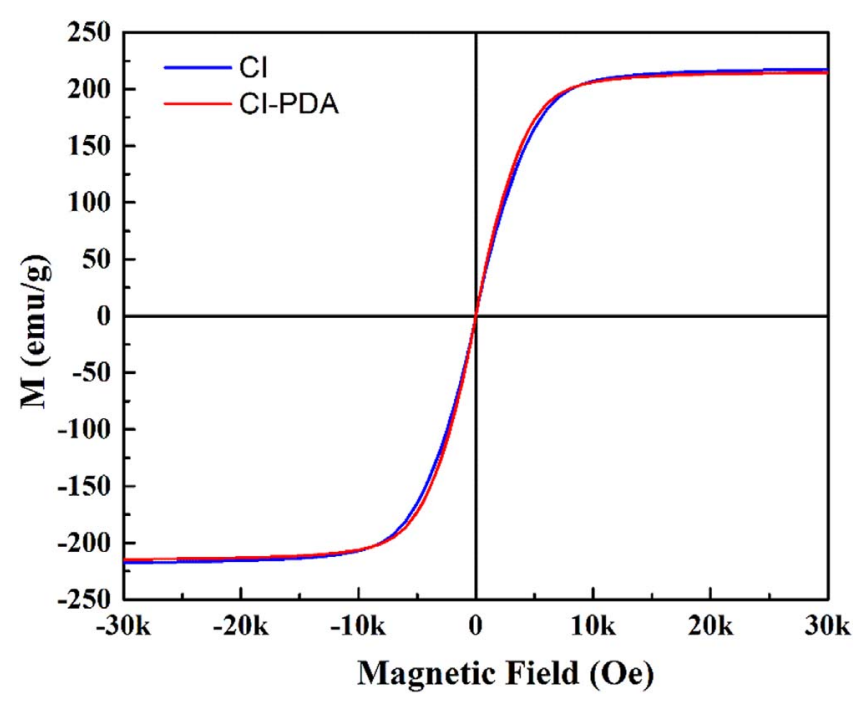

Figure 4. The $M-H$ curves of untreated CI and CI-PDA particles.

super paramagnetic behavior for both CI and CI-PDA [29]. The saturation magnetization value $(M s)$ of pure CI particles and CI-PDA particles were $217.3 \mathrm{emu} \cdot \mathrm{g}^{-1}$ and $214.6 \mathrm{emu} \cdot \mathrm{g}^{-1}$, respectively. This suggests the PDA surface modification had not resulted in any significant change in the magnetic properties of CI particles.

\subsection{Microstructure and mechanical properties of MREs}

The SEM images and the corresponding EDS mapping of $\mathrm{Fe}$ element for SR/CI and SR/CI-PDA particles are displayed in figure 5. It can be seen that the CI and CI-PDA particles distributed anisotropically in the silicone rubber matrix and formed chain-like structure in the direction of the magnetic field applied during the curing process.

The mechanical properties of pure SR and containing both treated and untreated magnetic particles were investigated. Figure 6(a) depicts the typical stress-strain curves for SR, SR/CI and SR/CI-PDA and the corresponding tensile strength, elongation at break and elastic modulus for each material are shown in figure 6(b). It can be seen that the incorporation of both CI and CI-PDA particles decreased the tensile strength of pure SR. When compared with SR/CI composites, both tensile strength and elongation at break of SR/CI-PDA composites were improved. In particular, the elongation at break of SR/CI-PDA equalled that of pure SR. This indicates that the interaction between the particles and the silicone rubber was enhanced after surface modification of CI particles. Generally, the incorporation of various inorganic fillers into a polymer matrix leads to a sharp increase in the elastic modulus due to the stiffening effect of the fillers. In this work, the same phenomenon was also observed and hence figure 6(b) shows that the elastic moduli of the composites containing CI or CI-PDA particles were higher than that of pure SR. However, the elastic moduli of MREs filled with CI-PDA particles were lower than those of the MREs filled with CI particles. Figure 6(a) shows that the composites containing particles did not conform to the typical uniaxial load/displacement curve associated with elastomers. The curve did not tend to an ' $\mathrm{S}$ ' configuration as did that for the unfilled SR. This was particularly true for the composite containing untreated CI where the influence of the particles was to stiffen the material in the stress-strain range below the region where crosslinks broke down. Notably, the SR/CI composite failed at a much lower elongation than the SR and SR/CI-PDA composites. The SR/CI-PDA composites did not exhibit this brittleness, in fact they achieved slightly higher elongations at failure than the unfilled SR samples. Hence, it is reasonable to conclude that the polydopamine coated CI particles, having less severe transitions in modulus and Poisson's ratio at the interfacial layers, allow higher strains for given stresses.

\subsection{Magnetorheological properties of MREs}

3.3.1. Magnetic field induced viscoelastic properties of MREs. Firstly, the effect of a magnetic field on the viscoelastic properties of MREs was investigated. The measurements were made from tests carried out at a constant strain amplitude of $0.1 \%$ and a frequency of $10 \mathrm{~Hz}$. In successive tests, the electric current was increased from 0 to $5 \mathrm{~A}$ which corresponds to a magnetic flux density of 0 to $1088 \mathrm{mT}$. Figure 7 shows the magnetic-dependent shear storage modulus $\left(G^{\prime}\right)$, loss modulus $\left(G^{\prime \prime}\right)$ and loss factor (tan $\delta$ ) for pure SR and MREs containing CI and CI-PDA particles. The storage modulus and loss modulus of pure SR remained unchanged with increasing magnetic flux density and was lower than the zero-field storage modulus and loss modulus of SR/CI and SR/CI-PDA composites (figures 7(a) and (b)). From figure 7(a) it can be seen that for both SR/CI and SR/CI-PDA composites, the shear storage modulus increased significantly with increasing magnetic flux density up to $600 \mathrm{mT}$ and thereafter leveled off. This phenomenon has been observed previously in similar tests on MREs $[13,30,32,39]$. This is because under the application of an external magnetic field, the enhanced particle magnetization and magnetic interactions between the particles reinforced the composite structure and resulted in a field responsive modulus [30]. However, when the magnetic flux density exceeded 600 $\mathrm{mT}$, the particle magnetization reached saturation and hence only minor changes in storage modulus were induced.

The dependence of loss modulus on magnetic flux density exhibited a similar trend, with a substantial rise as magnetic flux density increased and then reached a stable value as shown in figure 7(b). The increase in the loss modulus with increasing magnetic field was mainly due to the large energy dissipation caused by friction between the magnetic particles as they were rearranged under the applied magnetic field. The possible rupture of the matrix polymer segments during the re-structuring of the magnetic particles could also have contributed to the increases in loss modulus [40]. As for the dependence of loss modulus on magnetic flux density, when the magnetic field was sufficiently strong (in excess of $600 \mathrm{mT}$ ), the particle magnetization reached a saturation and consequently resulted in an almost unchanged loss modulus in the materials for further increases in magnetic flux density. 

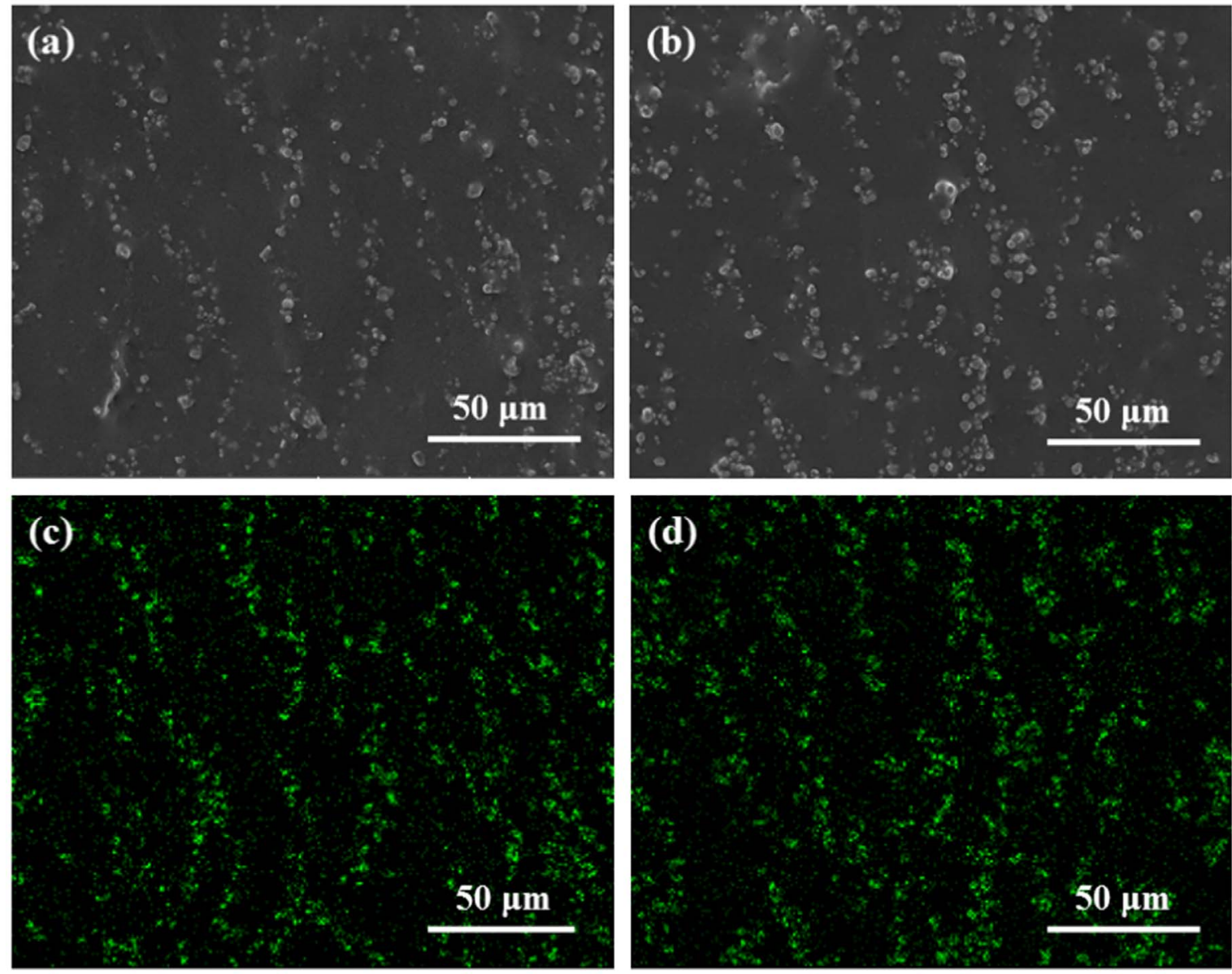

Figure 5. SEM images of (a) SR/CI and (b) SR/CI-PDA; EDS mapping of Fe element for (c) SR/CI and (d) SR/CI-PDA.
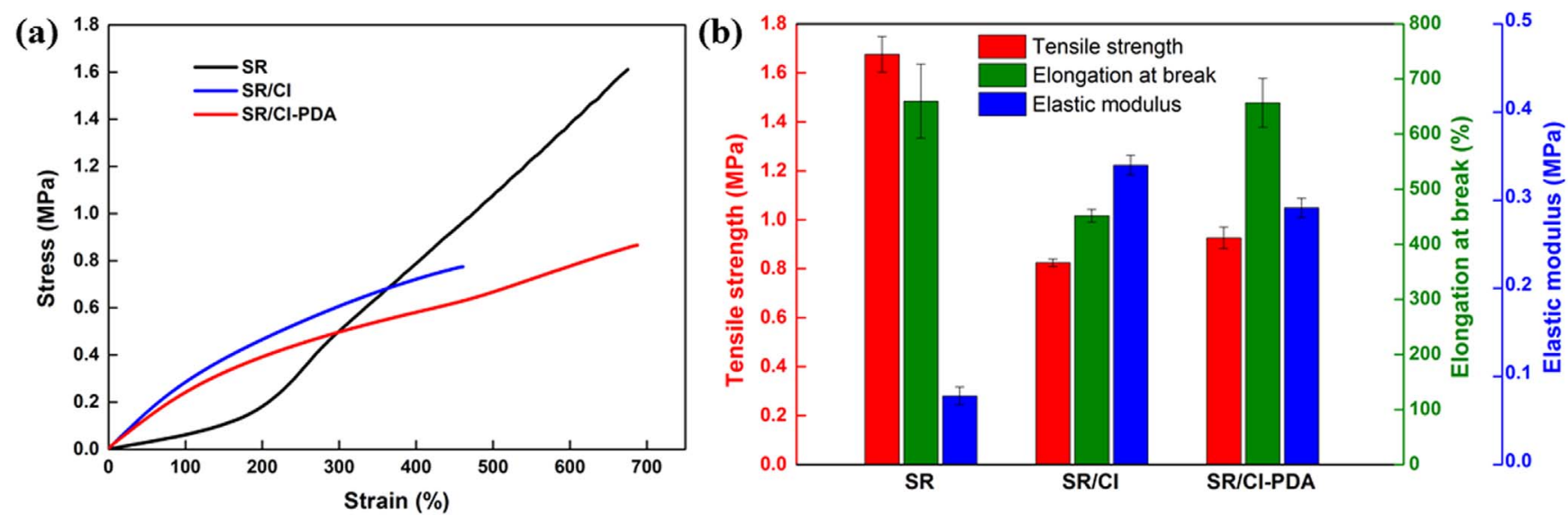

Figure 6. (a) Typical stress-strain curves and (b) tensile strength, elongation at break and elastic modulus for SR, SR/CI and SR/CI-PDA.

Furthermore, the effect of PDA modification on the viscoelastic properties of MREs was evaluated. It can be seen from figures 7(a) and (b) that the zero-field storage modulus and loss modulus of SR/CI-PDA was slightly lower than that of SR/CI. The shear modulus of MREs in zero fields can be calculated by using the Einstein-Guth-Gold equation [41]:

$$
G^{\prime}=G_{0}\left(1+2.5 \Phi+14.1 \Phi^{2}\right)
$$

where $G^{\prime}$ is the shear storage modulus of MRE, $G_{0}$ is the shear modulus of the unfilled matrix and $\Phi$ is the volume fraction of particles. The PDA coating resulted in a decreased volume fraction for the CI particles in SR/CI-PDA composites by comparison with that for SR/CI composites and the CI-PDA partially displayed polymeric mechanical behavior, so the zero-field shear modulus of SR/CI-PDA was lower than that of SR/CI. However, the shear storage 

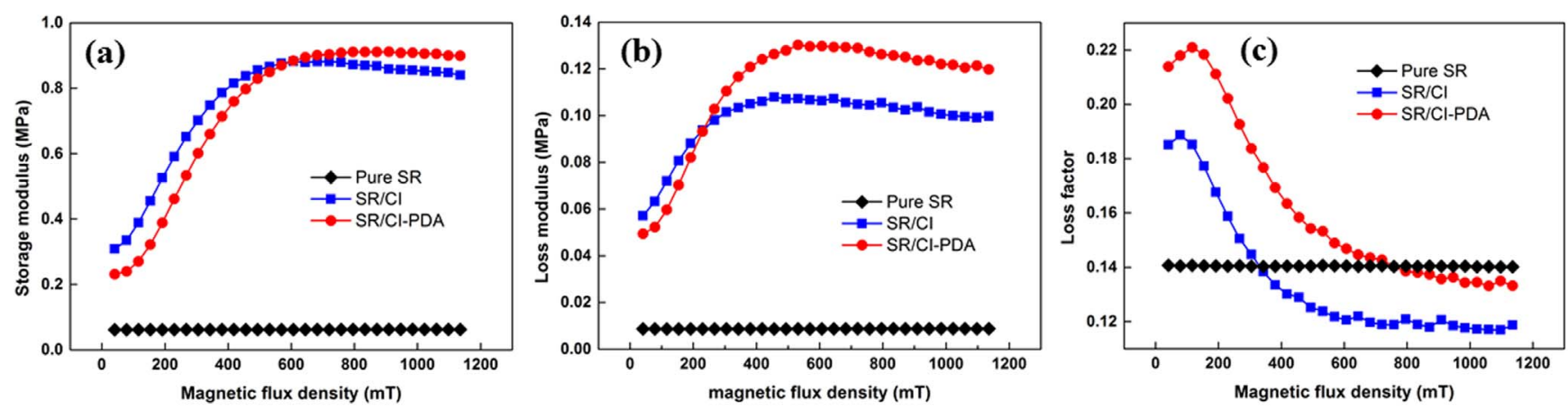

Figure 7. Dependence of (a) shear storage modulus, (b) shear loss modulus and (c) loss factor on the applied magnetic flux density for pure SR, SR/CI and SR/CI-PDA.

Table 1. Field-off/on $G^{\prime}$ of SR/CI and SR/CI-PDA and their MR effect.

\begin{tabular}{lcccc}
\hline Sample & $G_{0}^{\prime}(\mathrm{MPa})$ & $G^{\prime}{ }_{H}(\mathrm{MPa})$ & $\Delta G^{\prime}(\mathrm{MPa})$ & $e(\%)$ \\
\hline SR/CI & $0.31 \pm 0.005$ & $0.88 \pm 0.007$ & $0.57 \pm 0.002$ & $187 \pm 2.78$ \\
SR/CI-PDA & $0.23 \pm 0.004$ & $0.91 \pm 0.005$ & $0.68 \pm 0.002$ & $294 \pm 4.11$ \\
\hline
\end{tabular}

modulus of SR/CI-PDA was higher than that of SR/CI at the point where magnetization saturation was reached. This was because the bonding force between the CI particles and the silicone rubber improved after PDA modification, with the magnetic field induced interaction between the particles transferring effectively to the matrix and hence resulting in a greater increase in the storage modulus [28]. The stronger structure formed in the SR/CI-PDA composite under a magnetic field required more energy to rupture the material, which resulted in a higher loss modulus than that of SR/CI (figure 7(b)).

Figure 7(c) illustrates the dependence of loss factor (tan $\delta$ ) on magnetic flux density. It is shown that with the increase of the external magnetic field, the damping loss factor of both $\mathrm{SR} / \mathrm{CI}$ and SR/CI-PDA composites was raised to a maximum at a flux density of about $100 \mathrm{mT}$. Thereafter, it gradually fell to a steady value with further increases in magnetic flux density. It is generally known that the overall damping capacity of MREs is a combination of the intrinsic damping determined by the filler content and the constitutive damping capacity of each individual component, the interface damping caused by the internal friction between the two material phases and the magneto-mechanical damping due to the occurrence of magnetic loss [42]. When a low magnetic field was applied to the MRE samples, the magnetic force between the magnetic particles induced an increase in the magnetic interaction between the particle and the matrix. This led to more energy dissipated due to the interface slippage and the higher damping loss factor of the MREs. With further increases in the magnetic field, the rubber was more constrained due to the increased interaction between the magnetic particles. Therefore, the energy dissipation at the interface decreased and the damping loss factor of the MREs declined accordingly [15, 43].

It can also be noted that the MREs with CI-PDA particles had higher loss factors than those containing CI particles, indicating an enhanced damping provided by the surface PDA modification of CI particles. The same phenomenon has been reported previously [44]. This is thought to be because the enhanced interfacial interaction between CI-PDA particles and the silicone rubber matrix resulted in a higher loss of energy due to the friction that occurs with the movement of particles relative to the surrounding matrix.

To compare the magnetorheological behaviour of SR/CI and SR/CI-PDA, the relative MR effect $(e)$ of the materials was evaluated from magneto-sweep measurements complying with equation (2):

$$
e=\frac{G_{H}^{\prime}-G_{0}^{\prime}}{G_{0}^{\prime}}
$$

where $G_{H}^{\prime}$ is the maximum field-on modulus and $G^{\prime}{ }_{0}$ is the field-off modulus. The corresponding results are summarized in table 1.

These results demonstrate that the relative MR effect of MREs containing PDA modified CI particles reached 294\%, an improvement of $57 \%$ on the MREs with pristine CI particles. The magneto-induced modulus, also known as absolute MR effect (given in equation (3)) was also enhanced for the SR/CI-PDA composites.

$$
\Delta G^{\prime}=G_{H}^{\prime}-G_{0}^{\prime}
$$

The higher MR effect of SR/CI-PDA composites than that of SR/CI composites could be attributed to two factors: firstly, the zero-field storage modulus of SR/CI-PDA composites was lower than that of SR/CI composites due to the partial polymeric mechanical behavior of the CI particles when they were coated with a PDA layer; secondly, the $-\mathrm{OH}$ and $-\mathrm{NH}$ groups in PDA could have formed hydrogen bonds with oxygen atoms in the silicone rubber backbone and hence improved the interfacial adhesion between CI particles and the SR matrix, consequently, the shear storage modulus of SR/CI-PDA was higher than that of $\mathrm{SR} / \mathrm{CI}$ at the point where magnetization saturation was 

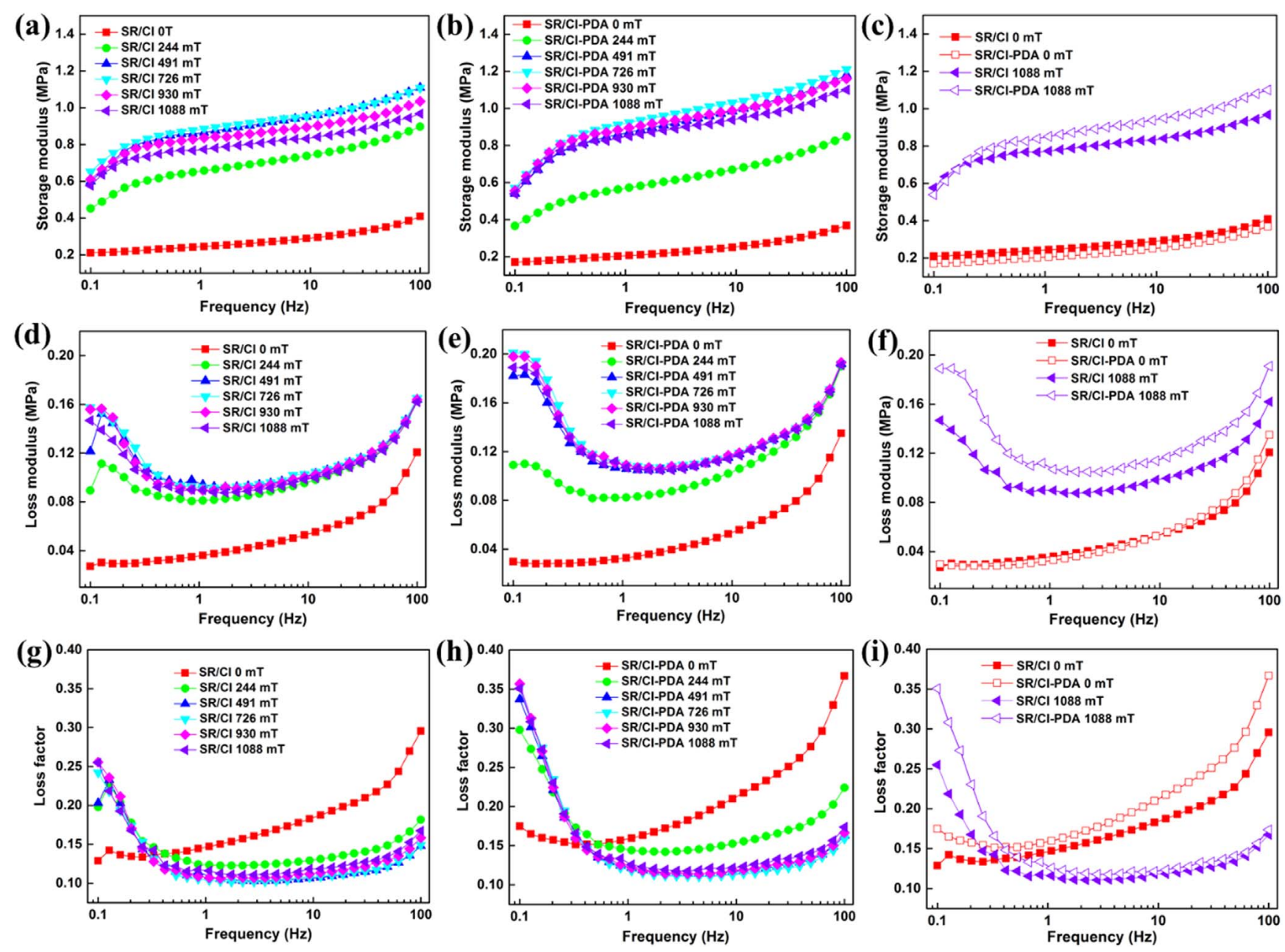

Figure 8. Dependence of shear storage modulus, loss modulus and loss factor on oscillation shear frequency under different magnetic flux densities for SR/CI ((a), (d), (g) respectively) and SR/CI-PDA ((b), (e), (h) respectively); comparison of the change of storage modulus (c), loss modulus (f) and loss factor (i) with frequency for SR/CI and SR/CI-PDA at magnetic flux densities of 0 and $1088 \mathrm{mT}$.

reached due to the magnetic field induced interaction between the particles transferred effectively to the matrix.

The results set out here indicate that the surface modification of CI particles provide MREs with not only improved mechanical properties but also enhanced magnetoinduced properties. Some cited research also found an increased MR effect after surface modification of magnetic particles [29, 30, 45], while others have suggested the opposite outcome [46]. This indicates that, though inconsistent, the surface modification of magnetic particles has significant influence on the MR properties of MRE composites. Selecting a suitable coating method and controlling the coating thickness is thus seen to be crucial when designing effective MREs with both improved mechanical properties and enhanced magneto-induced performance.

3.3.2. Frequency dependent viscoelastic properties. The frequency dependent viscoelastic properties of MREs was investigated. The strain amplitude was kept at $0.1 \%$ and the frequency was varied in the range of $0.1-100 \mathrm{~Hz}$ in all frequency tests. Figure 8 shows the change in storage modulus (figures 8(a) and (b)), loss modulus (figures 8(d) and (e)) and loss factor (figures $8(\mathrm{~g})$ and (h)) as a function of frequency at different magnetic flux densities for SR/CI and SR/CI-PDA composites. For direct comparison between SR/ CI and SR/CI-PDA, the change of storage modulus, loss modulus and loss factor with frequency of these two composites at zero magnetic field and at magnetic flux density of $1088 \mathrm{mT}$ are presented in figures 8(c), (f) and (i) respectively. As can be seen from figures 8(a) and (b), for a specific magnetic flux density, the storage modulus of both $\mathrm{SR} / \mathrm{CI}$ and SR/CI-PDA increased with increasing testing frequency. The same phenomenon has been reported in several publications [15, 29, 32, 33]. This is because when the frequency was increased, the deformation of the matrix molecular chain lagged behind the change in shear force and the dynamic response time of the samples decreased, which resulted in an increased stiffness for the whole system [15]. The zero-field storage modulus of SR/CI-PDA was lower than that of SR/CI and the magneto-induced storage modulus of SR/CI-PDA was higher than that of SR/CI in the whole frequency range (figure $8(\mathrm{c})$ ).

Figures $8(d)$ and (e) show that in the absence of a magnetic field, the loss modulus of both $\mathrm{SR} / \mathrm{CI}$ and SR/CI-PDA 

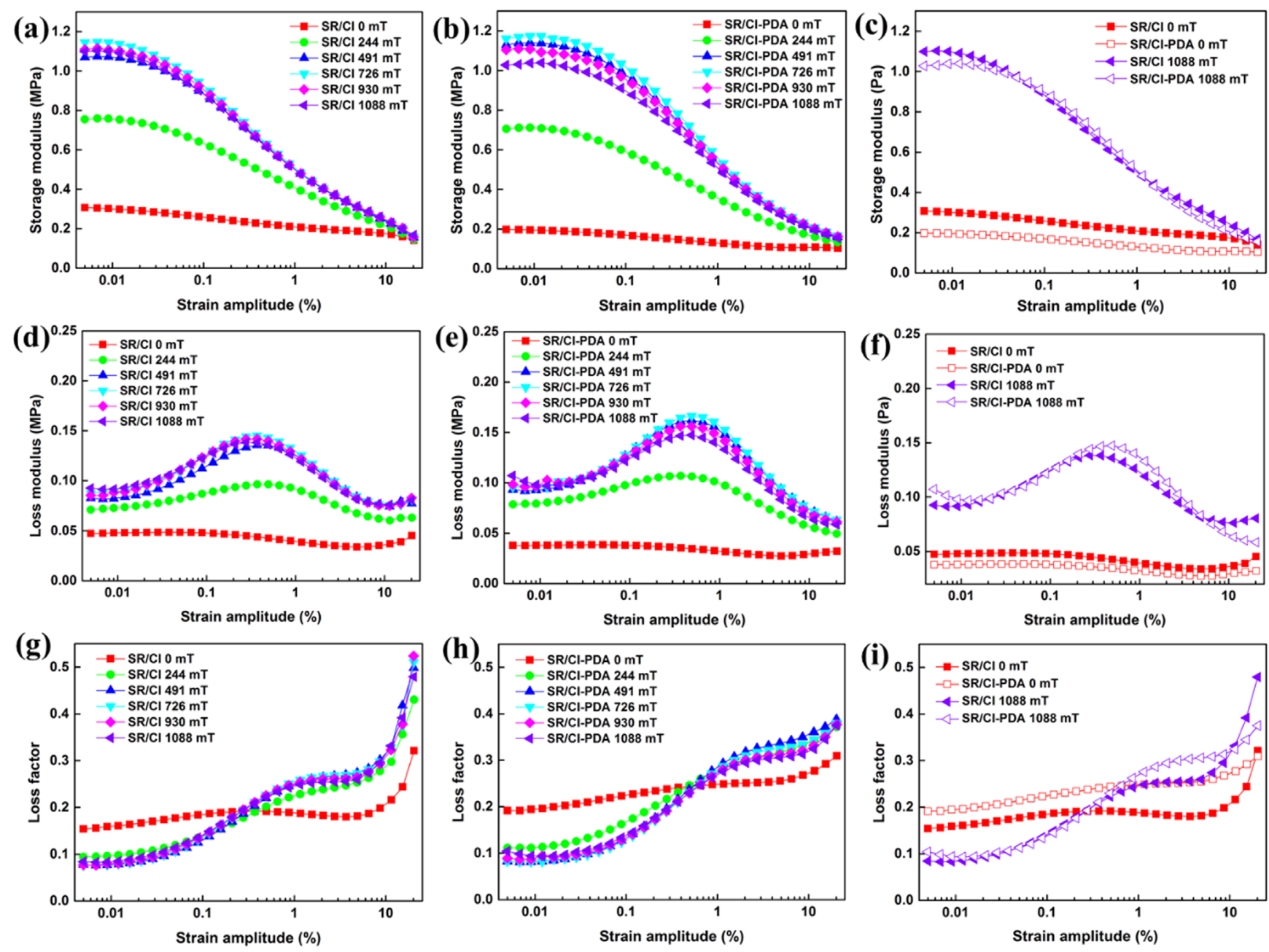

Figure 9. Dependence of shear storage modulus, loss modulus and loss factor on strain amplitude under different magnetic flux densities for SR/CI ((a), (d), (g) respectively) and SR/CI-PDA ((b), (e), (h) respectively); comparison of the change of storage modulus (c), loss modulus (f) and loss factor (i) with strain amplitude for SR/CI and SR/CI-PDA at magnetic flux densities of 0 and $1088 \mathrm{mT}$.

composites increased with increasing frequency. However, when the magnetic field was applied, the loss modulus for both SR/CI and SR/CI-PDA composites showed a decrease at low frequencies (of about $1 \mathrm{~Hz}$ ) and then increased with increasing frequency. The same trend was observed for the change of loss factor with increasing frequency (figures $8(\mathrm{~g})$ and $(\mathrm{h})$ ). Yu et al also reported this phenomenon in their work and ascribed this slight decrease in loss modulus in the initial stages to the friction transition from static friction at low frequency to dynamic friction at high frequency [29]. The loss modulus and loss factor of SR/CI-PDA in magnetic fields was higher than that of SR/CI due to greater energy loss in deformation for the SR/CI-PDA composites (figures 8(f) and (i)).

3.3.3. Strain-dependent viscoelastic property. Following frequency tests, the strain-dependent viscoelastic behavior of $\mathrm{SR} / \mathrm{CI}$ and SR/CI-PDA composites was evaluated. In the strain sweep experiments, the strain amplitudes were varied between $0.005 \%$ and $20 \%$ at a constant frequency of $10 \mathrm{~Hz}$. Figure 9 shows the change of storage modulus (figures 9 (a) and (b)), loss modulus (figures 9(d) and (e)) and loss factor (figures 9(g) and (h)) with strain amplitude at various magnetic flux densities for the SR/CI and SR/CI-PDA composites. For direct comparison between SR/CI and SR/CI-PDA, the change of storage modulus, loss modulus and loss factor with strain amplitude of these two composites at zero magnetic field and at magnetic flux density of $1088 \mathrm{mT}$ are presented in figures 9(c), (f) and (i) respectively. Figures 9(a) and (b) show that the storage modulus of both SR/CI and SR/CI-PDA decreased with the increasing strain amplitude, highlighting the characteristic strain softening effect also known as Payne effect. Furthermore, it was found that the Payne effect became more significant at higher magnetic flux densities. This is because magnetic interactions between the particles were stronger at higher magnetic flux densities, and the reduction in the magnetic interactions between magnetic particles was more pronounced with increasing strain [47]. As a consequence, the magnetic-induced storage modulus was reduced with increasing strain amplitude. It also should be noted that the magneto-induced Payne effect was more marked for SR/CI-PDA because of the lower zero-field storage modulus of SR/CI-PDA (figure 9(c)) as described earlier.

Figures 9(d) and (e) show the change of loss modulus with increasing strain amplitude for SR/CI and SR/CI-PDA respectively. It can be seen that the loss modulus of both $\mathrm{SR} /$ 
$\mathrm{CI}$ and SR/CI-PDA increased with increases in strain at small strain amplitudes. This can be explained by the greater energy needed for continuous rupture due to the magnetic interaction between particles with increasing strain amplitude. When the strain amplitude was further increased, the Payne effect caused a significant decrease in loss modulus, primarily because of the reduced particle interactions owing to the increased particle distances under high strain.

The change of loss factor with increasing strain amplitudes for SR/CI and SR/CI-PDA is depicted in figures $9(\mathrm{~g})$ and $(\mathrm{h})$ respectively. In the absence of a magnetic field, the loss factor increased slightly as the strain amplitude increased. However, the change of loss factor in the presence of a magnetic field was dramatically different. We can see that the effect of strain amplitude on loss factor was more pronounced than when a magnetic field was absent for both SR/CI and SR/CI-PDA. This is because the application of a magnetic field caused the restructuring of magnetic fillers and the formation of magnetic couplings between magnetic particles which are strong enough to resist breakage at small strain amplitudes [48]. Hence, the energy dissipation was reduced and the loss factor decreased compared with values obtained in the absence of magnetic field. With increasing strain amplitude, the breaking of the magnetic coupling demanded energy and resulted in an increased loss factor. The increase of loss factor with increasing strain amplitude in the range of $0.005 \%-20 \%$ was more pronounced for SR/CI than for SR/CI-PDA (figure 9(i)). This is because the improved particle-matrix interactions in SR/CI-PDA restricted the relative interface movement of $\mathrm{CI}$ particles at higher strain amplitude, which reduced the energy dissipation and consequently led to an indistinct increase of loss factor with increasing strain.

\section{Conclusions}

The surface of carbonyl iron particles was modified by employing bio-inspired polydopamine adaptation and the modified particles were used to fabricate MRE composites which exhibited an enhanced magnetorheological effect. The morphology and influence of surface modification on the viscoelastic properties of the silicone rubber based MREs were evaluated. It was found that a thin layer of polydopamine was successfully coated onto the surface of carbonyl iron particles to an approximate thickness of $27.5 \mathrm{~nm}$. Whilst continuing to maintain the intrinsic good magnetic properties of carbonyl iron particles, polydopamine modification resulted in much improved mechanical properties including gains in tensile strength and elongation at break for the MREs. The MREs containing PDA modified CI particles showed a lower initial (zero-field) storage modulus than that of MREs with untreated CI particles, but attained a higher storage modulus when the magnetization saturation was reached, resulting in enhancement of both absolute and relative magnetorheological effects in the MREs. With both improved mechanical properties and enhanced magnetic field induced changes in modulus, the MREs containing polydopamine modified CI particles present the possibility of wider applications and greater durability for SR based MREs.

\section{Acknowledgments}

The authors would like to give thanks to the financial support from the Shandong Provincial Key Research and Development Program, China (Grant no. 2019GGX102071), the National Natural Science Foundation of China (Grant no. 51703108), the Shandong Provincial Natural Science Foundation, China (Grant no. ZR2017BEM042) and the Qingdao Postdoctoral Applied Basic Research Project. The dynamic viscoelastic property testing provided by Professor Jiong Wang of Nanjing University of Science and Technology are also greatly appreciated.

\section{ORCID iDs}

Liang Jiang (ib https://orcid.org/0000-0002-7792-6069

\section{References}

[1] Cvek M, Kracalik M, Sedlacik M, Mrlik M and Sedlarik V 2019 Reprocessing of injection-molded magnetorheological elastomers based on TPE matrix Composites B 172 253-61

[2] Sun S S, Yildirim T, Wu J, Yang J, Du H, Zhang S W and Li W H 2017 Design and verification of a hybrid nonlinear MRE vibration absorber for controllable broadband performance Smart Mater. Struct. 269

[3] Liu G Y, Lu K, Zou D L, Xie Z L, Rao Z S and Ta N 2017 Development of a semi-active dynamic vibration absorber for longitudinal vibration of propulsion shaft system based on magnetorheological elastomer Smart Mater. Struct. 2616

[4] Chen D, Yu M, Zhu M, Qi S and Fu J 2016 Carbonyl iron powder surface modification of magnetorheological elastomers for vibration absorbing application Smart Mater. Struct. 259

[5] Kozlowska J, Boczkowska A, Czulak A, Przybyszewski B, Holeczek K, Stanik R and Gude M 2016 Novel MRE/CFRP sandwich structures for adaptive vibration control Smart Mater. Struct. 2514

[6] Wang Q, Dong X F, Li L Y and Ou J P 2017 Study on an improved variable stiffness tuned mass damper based on conical magnetorheological elastomer isolators Smart Mater. Struct. 2610

[7] Qi S, Guo H, Chen J, Fu J, Hu C, Yu M and Wang Z L 2018 Magnetorheological elastomers enabled high-sensitive selfpowered tribo-sensor for magnetic field detection Nanoscale 10 4745-52

[8] Shabdin M K et al 2019 Material characterizations of Gr-based magnetorheological elastomer for possible sensor applications: rheological and resistivity properties Materials 1215

[9] Tong Y, Dong X and Qi M 2018 Improved tunable range of the field-induced storage modulus by using flower-like particles as the active phase of magnetorheological elastomers Soft Matter 14 3504-9 
[10] Shuib R K, Pickering K L and Mace B R 2015 Dynamic properties of magnetorheological elastomers based on iron sand and natural rubber J. Appl. Polym. Sci. 13241506

[11] Galipeau E and Ponte Castañeda P 2012 The effect of particle shape and distribution on the macroscopic behavior of magnetoelastic composites Int. J. Solids Struct. 49 1-17

[12] Zhou Y, Jerrams S, Betts A, Farrell G and Chen L 2015 The influence of particle content on the equi-biaxial fatigue behaviour of magnetorheological elastomers Mater. Des. 67 398-404

[13] Yao J, Yang W, Gao Y, Scarpa F and Li Y 2019 Magnetorheological elastomers with particle chain orientation: modelling and experiments Smart Mater. Struct. 28095008

[14] Samal S, Skodova M and Blanco I 2019 Effects of filler distribution on magnetorheological silicon-based composites Materials 1213

[15] Khairi M H A, Abd Fatah A Y, Mazlan S A, Ubaidillah U, Nordin N A, Ismail N I N, Choi S B and Aziz S A A 2019 Enhancement of particle alignment using silicone oil plasticizer and its effects on the field-dependent properties of magnetorheological elastomers Int. J. Mol. Sci. 2015

[16] Boczkowska A, Awietjan S F, Pietrzko S and Kurzydłowski K J 2012 Mechanical properties of magnetorheological elastomers under shear deformation Composites B $\mathbf{4 3}$ 636-40

[17] Yu M T, Qi S, Fu J, Yang P A and Zhu M 2015 Preparation and characterization of a novel magnetorheological elastomer based on polyurethane/epoxy resin IPNs matrix Smart Mater. Struct. 248

[18] Wang Y H, Zhang X R, Oh J and Chung K 2015 Fabrication and properties of magnetorheological elastomers based on CR/ENR self-crosslinking blends Smart Mater. Struct. 2412

[19] Zając P, Kaleta J, Lewandowski D and Gasperowicz A 2010 Isotropic magnetorheological elastomers with thermoplastic matrices: structure, damping properties and testing Smart Mater. Struct. 19045014

[20] Zhou Y F, Johnson M, Wen S P, Betts A and Jerrams S 2017 Equi-biaxial fatigue behaviour of magnetorheological elastomers in magnetic fields J. Intell. Mater. Syst. Struct. 28 687-96

[21] Bica I, Anitas E M, Averis L M E, Kwon S H and Choi H J 2019 Magnetostrictive and viscoelastic characteristics of polyurethane-based magnetorheological elastomer $J$. Ind. Eng. Chem. 73 128-33

[22] Asadi Khanouki M, Sedaghati R and Hemmatian M 2019 Experimental characterization and microscale modeling of isotropic and anisotropic magnetorheological elastomers Composites B 176107311

[23] Chen L, Gong X L, Jiang W Q, Yao J J, Deng H X and Li W H 2007 Investigation on magnetorheological elastomers based on natural rubber J. Mater. Sci. 42 5483-9

[24] Gorman D, Murphy N, Ekins R and Jerrams S 2017 The evaluation of the effect of strain limits on the physical properties of magnetorheological elastomers subjected to uniaxial and biaxial cyclic testing Int. J. Fatigue 103 1-4

[25] Aloui S and Klüppel M 2015 Magneto-rheological response of elastomer composites with hybrid-magnetic fillers Smart Mater. Struct. 24025016

[26] Qi S, Yu M, Fu J, Zhu M, Xie Y and Li W 2018 An EPDM/ MVQ polymer blend based magnetorheological elastomer with good thermostability and mechanical performance Soft Matter 14 8521-8

[27] Damiani R and Sun L Z 2017 Microstructural characterization and effective viscoelastic behavior of magnetorheological elastomers with varying acetone contents Int. J. Damage Mech. 26 103-17
[28] Pickering K L, Raa Khimi S and Ilanko S 2015 The effect of silane coupling agent on iron sand for use in magnetorheological elastomers 1: surface chemical modification and characterization Composites A 68 377-86

[29] Yu M, Qi S, Fu J, Zhu M and Chen D 2017 Understanding the reinforcing behaviors of polyaniline-modified carbonyl iron particles in magnetorheological elastomer based on polyurethane/epoxy resin IPNs matrix Compos. Sci. Technol. 139 36-46

[30] Cvek M, Mrlík M, Ilčíková M, Mosnáček J, Münster L and Pavlínek V 2017 Synthesis of silicone elastomers containing silyl-based polymer-grafted carbonyl iron particles: an efficient way to improve magnetorheological, damping, and sensing performances Macromolecules 50 2189-200

[31] Kwon S H, An J S, Choi S Y, Chung K H and Choi H J 2019 Poly(glycidyl methacrylate) coated soft-magnetic carbonyl iron/silicone rubber composite elastomer and its magnetorheology Macromol. Res. 27 448-53

[32] Raa Khimi S and Pickering K L 2016 The effect of silane coupling agent on the dynamic mechanical properties of iron sand/ natural rubber magnetorheological elastomers Composites B 90 115-25

[33] An J S, Kwon S H, Choi H J, Jung J H and Kim Y G 2017 Modified silane-coated carbonyl iron/natural rubber composite elastomer and its magnetorheological performance Compos. Struct. 160 1020-6

[34] Lee H, Dellatore S M, Miller W M and Messersmith P B 2007 Mussel-Inspired surface chemistry for multifunctional coatings Science 318 426-30

[35] Jiang L, Kennedy D, Jerrams S and Betts A 2016 Enhancement of dielectric properties with the addition of bromine and dopamine modified barium titanate particles to silicone rubber MRS Commun. 6 437-41

[36] Knorr D B, Tran N T, Gaskell K J, Orlicki J A, Woicik J C, Jaye C, Fischer D A and Lenhart J L 2016 Synthesis and characterization of aminopropyltriethoxysilanepolydopamine coatings Langmuir 32 4370-81

[37] Li X, Shan H, Cao M and Li B 2018 Mussel-inspired modification of PTFE membranes in a miscible THF-Tris buffer mixture for oil-in-water emulsions separation J. Membr. Sci. 555 237-49

[38] Yang D, Tian M, Li D, Wang W, Ge F and Zhang L 2013 Enhanced dielectric properties and actuated strain of elastomer composites with dopamine-induced surface functionalization J. Mater. Chem. A 1 12276-84

[39] Kwon S H, Lee C J, Choi H J, Chung K H and Jung J H 2019 Viscoelastic and mechanical behaviors of magnetorheological carbonyl iron/natural rubber composites with magnetic iron oxide nanoparticle Smart Mater. Struct. 288

[40] Sorokin V V, Stepanov G V, Shamonin M, Monkman G J, Khokhlov A R and Kramarenko E Y 2015 Hysteresis of the viscoelastic properties and the normal force in magnetically and mechanically soft magnetoactive elastomers: effects of filler composition, strain amplitude and magnetic field Polymer 76 191-202

[41] Guth E 1945 Theory of filler reinforcement J. Appl. Phys. 16 20-5

[42] Yang J, Gong X L, Deng H X, Qin L J and Xuan S H 2012 Investigation on the mechanism of damping behavior of magnetorheological elastomers Smart Mater. Struct. 21 125015

[43] Chen L, Gong X L and Li W H 2008 Damping of magnetorheological elastomers Chin. J. Chem. Phys. 21 $581-5$

[44] Ge L, Gong X, Wang Y and Xuan S 2016 The conductive three dimensional topological structure enhanced magnetorheological elastomer towards a strain sensor Compos. Sci. Technol. 135 92-9 
[45] Qiao X Y, Lu X S, Li W H, Chen J, Gong X L, Yang T, Li W, Sun K and Chen X D 2012 Microstructure and magnetorheological properties of the thermoplastic magnetorheological elastomer composites containing modified carbonyl iron particles and poly(styrene-bethylene-ethylenepropylene-b-styrene) matrix Smart Mater Struct. 21115028

[46] Li J F, Gong X L, Zhu H and Jiang W Q 2009 Influence of particle coating on dynamic mechanical behaviors of magnetorheological elastomers Polym. Test. 28 331-7
[47] Sorokin V V, Stepanov G V, Shamonin M,

Monkman G J and Kramarenko E Y 2017

Magnetorheological behavior of magnetoactive elastomers filled with bimodal iron and magnetite particles Smart Mater. Struct. 2612

[48] Sorokin V V, Ecker E, Stepanov G V, Shamonin M, Monkman G J, Kramarenko E Y and Khokhlov A R 2014 Experimental study of the magnetic field enhanced Payne effect in magnetorheological elastomers Soft Matter 10 8765-76 